\title{
Percepción del feminismo en mujeres y hombres de la ciudad de Cuenca, Ecuador
}

\section{Perception of feminism in women and men in the city of Cuenca, Ecuador}

\author{
Cristina Sacaquirin-Rivadeneira ${ }^{10}$, Eva Peña-Contreras \\ Facultad de Psicología, Universidad de Cuenca. Cuenca, Ecuador. \\ *Autor para correspondencia: cris_95@live.com \\ Fecha de recepción: 18 de septiembre de 2020 - Fecha de aceptación: 2 de noviembre de 2020
}

\section{RESUMEN}

El presente estudio explora las percepciones frente al feminismo tanto de mujeres y de hombres residentes en la ciudad de Cuenca, Ecuador, mediante la participación en grupos focales. En total se conformaron cinco grupos focales con 25 participantes (hombres y mujeres) en edades comprendidas entre los 18 y 60 años. Los datos fueron analizados mediante el programa Atlas.ti utilizando técnicas de codificación inductiva. De las narrativas recopiladas en este estudio se concluyó que, el feminismo se percibe desde una esfera colectiva e individual y, si bien ha logrado posicionar su lucha en torno al reconocimiento de los derechos de las mujeres, también se evidencian a su alrededor connotaciones y estereotipos de carácter negativo que se perciben influenciadas por diversos factores que responden a un contexto sociocultural específico. No obstante, se reivindicó el objetivo feminista en busca de la igualdad de derechos en las estructuras y prácticas sociales y se resaltó la necesidad del movimiento en la reeducación de las relaciones sociales de dominación y opresión tanto en el ámbito familiar como en el ámbito institucional.

Palabras clave: Feminismo, percepción, estereotipos, género.

\begin{abstract}
This study explores the perceptions of feminism both by women and men in the city of Cuenca in Ecuador through participation in focus groups. In total, five focus groups were formed with 25 participants between the ages of 18 and 60 years. The data were analyzed using the Atlas.ti program using inductive coding techniques. From the narratives compiled in this study, it is concluded that feminism is perceived from a collective and individual sphere and, although it has managed to position its struggle around the recognition of women's rights, negative connotations and stereotypes are also evident around it that are influenced by various factors that respond to a specific sociocultural context. However, the feminist objective that seeks equal rights in social structures and practices was vindicated and the need for the movement in the reeducation of social relations of domination and oppression both in the family sphere and in the institutional sphere was highlighted.
\end{abstract}

Keywords: Feminism, perception, stereotypes, gender.

\section{INTRODUCCIÓN}

Según Katzenstein (1987), el feminismo logra visualizar que las mujeres constituyen un grupo oprimido en comparación con los hombres. Se reconoce que esta condición estaría influenciada por procesos de desigualdad estructural más que por acciones o circunstancias individuales (Martin, 1990). Por lo tanto, el feminismo busca la transformación de las relaciones sociales basadas en la asimetría y la opresión tanto en la esfera pública como privada (García \& Valdivieso, 2005), que lleven a alcanzar la liberación de la mujer como del hombre (Gamba, 2007). Y para que esta visión ocurra el cambio tiene que ser social, político, económico y cultural (Mueller, 1987).
Un aspecto que ha tomado relevancia al momento de hablar de feminismo radica en la interseccionalidad (Valentine, 2007). Esta hace referencia a que cada individuo (en este caso, la mujer) se circunscribe, en base a diferentes categorías sociales (p.ej., raza), dentro de estructuras más amplias y complejas de desigualdad (Collins, 2000), variando en relevancia y notoriedad en los diferentes contextos sociales (Hopkins, 2017). Se ha enfatizado que el feminismo no constituye un dogma o una ideología (Jaggar, 1983). Por el contrario, se ha ido configurando en distintos movimientos sociales que, a partir de su intervención temporal concreta se sitúan en permanente confrontación y diálogo con la realidad social, además de su propia evolución interna (Montero, 2006). 
Con respecto a esta diversificación, se debe vislumbrar (más allá de sus diferencias) que estos feminismos se enmarcan en un mismo objetivo. El movimiento de feministas liberales busca la igualdad de oportunidades y acceso al mercado laboral. Por su parte, el feminismo radical establece que la opresión de la mujer es la raíz que configura las formas de opresión y de dominación (patriarcado). El feminismo socialista argumenta que la liberación de la mujer sólo se logrará a partir de las transformaciones de las relaciones sociales y de las fuerzas productivas, incluyendo la actividad laboral que las mujeres realizan en el hogar (cf. Friedman et al., 1987). Por último, los movimientos ecológicos se debaten, por una parte, entre revalorizar la heterodesignación histórica de la mujer y, por el contrario, otros critican y rechazan el esencialismo en esta concepción que resulta androcéntrica y heteropatriarcal (Anzoátegui, 2019).

En torno a la filiación, aceptación o rechazo de las diferentes propuestas feministas, la teoría de la identidad social resalta que las personas están más dispuestas a identificarse con un grupo si este es evaluado positivamente (Breen \& Karpinski, 2008). Por su parte, Hemmings (2012) argumenta que a través de la disonancia afectiva (discrepancia entre la narración propia vs. la realidad social) se podría (o no) desarrollar un sentido de empatía (presente en mujeres y hombres) y la voluntad de participar colectivamente del movimiento feminista.

Cuando una persona o un grupo de personas se hacen llamar feministas, esta etiqueta, refleja en la sociedad la presencia de estereotipos negativos y positivos con diferentes implicaciones (Dyer \& Hurd, 2016). En la literatura se hace referencia al continuo feminista para describir las distintas articulaciones que tienen lugar al asumir el feminismo a nivel personal, estas incluyen: (a) personas que se identifican como feministas (soy feminista), (b) aquellas que se identifican como feministas pero explicitan un grado de desacuerdo (soy feminista, pero), (c) a personas que no se identifican con el movimiento pero poseen actitudes feministas (no soy feminista, pero), y (d) a quienes no se identifican como feministas y tampoco están de acuerdo con los objetivos feministas (no soy feminista; Jolles, 2012; Butler, 2013).

No obstante, en la actualidad, ante lo que parece percibirse como una multiplicidad de discursos con implicaciones teóricas (p.ej., ¿qué es feminismo? vs. ¿qué no es feminismo?), más la distorsión de la información en los medios de comunicación, en las redes y la intolerancia social en nuestro contexto (p.ej., Díaz, 08 de marzo de 2018; Muñoz, 15 de mayo de 2018), el feminismo continúa enfrentando la misma reticencia paradójica y estigmatización (Hoskin et al., 2017). Los movimientos feministas se enfrentan al reto de cómo comunicar y sostener la reivindicación de los derechos asentada en una legitimidad real y no formal (Gifford, 2011), además de preservar lo que ya se ha logrado (Valdivieso et al., 2012).

Sin embargo, para lograr esa igualdad son relevantes las políticas de Estado y su aplicación real con criterios de efectividad. En nuestro país, actualmente, estas se establecen bajo las premisas del Plan Nacional para el Desarrollo "Toda una Vida" (Secretaría Nacional de Planificación y Desarrollo, 2017), enmarcado en los compromisos internacionales de desarrollo global y en sus objetivos de Desarrollo Sostenible (Agenda 2030; Organización de las Naciones Unidas [ONU], 2015). Dentro de estos planteamientos se propone alcanzar la igualdad de género y la garantía plena de los derechos de las mujeres en todas sus áreas.

En este sentido, es necesario problematizar el papel del Estado como supuesto garante de los derechos de las mujeres. Es evidente el abismo entre las propuestas legislativas y un cambio real (cf. Pardo, 2019). Un claro ejemplo de la ineficacia del Estado ecuatoriano, en un paso más para materializar esa igualdad, supone el veto total al Código Orgánico de Salud (COS; Asamblea Nacional de la República del Ecuador, 2020), en donde se abordaban temas de derechos sexuales y reproductivos de las mujeres y su atención integral.

A partir de este trasfondo y considerando las limitaciones metodológicas de investigaciones relacionadas, este estudio tuvo como objetivo el explorar las percepciones frente al feminismo tanto de mujeres y de hombres residentes en la ciudad de Cuenca - Ecuador. Lo que nos ha llevado a preguntarnos, ¿cómo perciben el feminismo las mujeres y los hombres en nuestro contexto?

\section{METODOLOGÍA}

Se utilizó un enfoque cualitativo transversal, mediante el uso de grupos focales como técnica de recopilación de información. Esta permitió la exploración de los constructos socioculturales que se configuran como una visión frente a un tema específico (Carey \& Asbury, 2012) y el análisis inductivo e interpretación a partir del discurso y la conducta observable de los participantes (Taylor \& Bodgan, 1992). Además, se consideró la perspectiva de género en el análisis de los datos descriptivos obtenidos (López et al., 2011).

El proceso se apoyó metodológicamente en los siguientes planteamientos: (a) la investigación es inductiva y no se comprueban hipótesis o teorías, (b) se asume una perspectiva holística del escenario y de las personas, (c) las investigadoras son sensibles a los efectos que puedan causar en los participantes, sin embargo, predomina la objetividad para no interferir en el discurso de los participantes, (d) no se busca la verdad sino la autenticidad, (e) se da un especial énfasis a la validez en la investigación y (f) se destaca el aspecto humano de la vida social y se acepta el error en el juicio humano (García, Alvira \& Alonso, 2015).

\subsection{Zona de estudio}

La selección de los participantes se realizó mediante un muestreo por conveniencia, intencional, en donde se seleccionan casos característicos de la población, limitando la muestra sólo a estos, y se procura que sea representativa de acuerdo con los objetivos del estudio (Scharager \& Reyes, 2001). En esta investigación, la representatividad se logró mediante la heterogeneidad de la muestra, al elegir personas de diversas áreas profesionales, académicas y sectores sociales, además de buscar números equitativos para la variable sexo. Para ello, el equipo de investigación sugirió nombres de participantes a los que se les consultaría su deseo de participar y personas que no estuviesen vinculadas profesionalmente a la Universidad de Cuenca (con excepción del grupo de expertas), con las condiciones especificadas en criterios de inclusión. 
Se consideraron los siguientes criterios de inclusión para los participantes: (a) hombres y mujeres de nacionalidad ecuatoriana residentes en la ciudad de Cuenca y, (b) que se encontraran en los siguientes rangos de edad, de $18 \mathrm{y}$ 19 años y de 20 a 60 años. Para la conformación de los grupos focales se consideraron los siguientes criterios: (a) se decidió que los grupos fueran homogéneos en base al sexo (mujeres y hombres, por separado), pero heterogéneos en cuanto a diversidad de actividades laborales o académicas y que no se conociesen entre sí con el fin de facilitar el intercambio de ideas y evitar que se realicen suposiciones o prejuicios (Díaz, 2005) y, (b) cada grupo focal estuvo conformado por cuatro a seis participantes (Onwuegbuzie et al., 2011).

En total se conformaron cinco grupos focales con 25 participantes. El primer grupo focal (con el que se realizó el pilotaje) estuvo conformado por cinco mujeres consideradas expertas en el tema, las mismas fueron contactadas por ser profesionales dedicadas al trabajo en temas de género de diferentes instituciones universitarias, así como activistas de grupos feministas de la cuidad. El segundo y tercer grupo focal estuvo conformado por cinco mujeres y cinco hombres (en cada grupo) de 18 y 19 años de edad. El cuarto grupo focal se conformó por seis mujeres de 21 a 27 años. Y en el quinto grupo focal participaron cuatro hombres de 21 a 39 años. Se consideraron algunos datos sociodemográficos de los participantes (Myers, 1998). Ver la Tabla 1 para la información específica.

\subsection{Instrumentos, procedimiento y análisis de datos}

Se diseñó una guía de grupo focal con preguntas semiestructuradas tomando como base las categorías teóricas identificadas en la revisión bibliográfica. Se incluyeron: (a) la definición de feminismo, (b) estereotipos en torno a las personas feministas, (c) connotación social frente al feminismo, (d) actitudes y pensamientos incompatibles con el feminismo y (e) la postura personal frente al feminismo. Esta guía fue discutida y aprobada dentro del equipo de investigación y posteriormente validada mediante una prueba piloto realizada con el grupo de expertas, de esta experiencia se realizaron ajustes a la entrevista original.

Para el registro de la información no verbal y el grado de consenso/desacuerdo, se elaboró una matriz en Word (Onwuegbuzie et al., 2011), la misma fue revisada y aprobada por el equipo de investigación.

La conformación de los grupos focales siguió las estrategias de reclutamiento de Beck et al. (2004) de tres pasos: (a) se enfatizó el interés en las opiniones de cada participante, los mismos fueron convocados con la consigna de discutir acerca de los distintos movimientos sociales; (b) la invitación se realizó de forma repetitiva, a través de llamadas o mensajes telefónicos, se coordinó previamente el horario, el lugar y se obtuvo la confirmación de su participación y (c) al final de cada grupo focal (modalidad física), las personas fueron

Tabla 1. Características sociodemográficas de participantes de los grupos focales.

\begin{tabular}{|c|c|c|c|c|c|c|c|}
\hline $\begin{array}{l}\text { Participante } \\
\text { (Seudónimo) }\end{array}$ & Sexo & Edad & Estado civil & $\begin{array}{c}\text { Nivel de } \\
\text { estudios } \\
\text { (culminado) }\end{array}$ & Ocupación & Religión & $\begin{array}{l}\text { Discapacidad/ } \\
\text { enfermedad } \\
\text { crónica }\end{array}$ \\
\hline Fernanda & Mujer & 19 & Soltera & Bachillerato & Estudiante & Católica & No \\
\hline Carmen & Mujer & 19 & Soltera & Bachillerato & Estudiante & Católica & No \\
\hline Laura & Mujer & 18 & Soltera & Bachillerato & Estudiante & Agnóstica & No \\
\hline Lucía & Mujer & 19 & Soltera & Bachillerato & Estudiante & Católica & No \\
\hline Adriana & Mujer & 18 & Soltera & Bachillerato & Estudiante & Católica & Sí \\
\hline Cecilia & Mujer & 22 & Soltera & Bachillerato & Estudiante & Católica & No \\
\hline Rosa & Mujer & 24 & Unión libre & Bachillerato & Estudiante & Cristiana & No \\
\hline Manuela & Mujer & 23 & Soltera & Pregrado & Ninguna & Ninguna & No \\
\hline Elena & Mujer & 27 & Soltera & Pregrado & Ninguna & Católica & No \\
\hline Matilde & Mujer & 26 & Soltera & Pregrado & Ninguna & Agnóstica & No \\
\hline Carlota & Mujer & 26 & Soltera & Pregrado & Docente & Católica & No \\
\hline Carlos & Hombre & 18 & Soltero & Bachillerato & Estudiante & Ninguna & No \\
\hline Luis & Hombre & 19 & Soltero & Bachillerato & Estudiante & Católico & No \\
\hline Hugo & Hombre & 18 & Soltero & Bachillerato & Estudiante & Católico & No \\
\hline Juan & Hombre & 19 & Soltero & Bachillerato & Ninguna & Católico & No \\
\hline Antonio & Hombre & 19 & Soltero & Bachillerato & Estudiante & Ninguna & No \\
\hline Alberto & Hombre & 39 & Soltero & Máster & Ninguna & Ateo & No \\
\hline Miguel & Hombre & 23 & Soltero & Bachillerato & Estudiante & Católico & No \\
\hline Víctor & Hombre & 25 & Soltero & Bachillerato & Estudiante & Católico & No \\
\hline Fernando & Hombre & 21 & Soltero & Bachillerato & Estudiante & Ninguna & No \\
\hline \multicolumn{8}{|c|}{ Grupo de expertas } \\
\hline Sofía & Mujer & 23 & Soltera & Bachillerato & Estudiante/ activista & Agnóstica & No \\
\hline Daniela & Mujer & 26 & Soltera & Pregrado & Estudiante/ activista & Agnóstica & No \\
\hline Emilia & Mujer & 33 & Divorciada & Máster & $\begin{array}{c}\text { Coordinadora } \\
\text { editorial/ activista }\end{array}$ & Agnóstica & Sí \\
\hline Camila & Mujer & 39 & Soltera & Doctorado & $\begin{array}{c}\text { Directora de género y } \\
\text { desarrollo } \\
\text { (universidad) }\end{array}$ & Agnóstica & No \\
\hline Paola & Mujer & 58 & Casada & Doctorado & $\begin{array}{l}\text { Directora de equidad y } \\
\text { género (universidad) }\end{array}$ & Católica & No \\
\hline
\end{tabular}


incentivadas por su participación mediante la entrega de un refrigerio.

Los grupos focales se realizaron en dos modalidades debido a la crisis sanitaria mundial causada por el covid19, lo que dificultó el encuentro físico. Tres grupos focales fueron realizados de forma presencial en el mes de febrero y dos se realizaron de forma virtual en los meses de abril y mayo (mediante el software Zoom). Previo al inicio de cada grupo focal, para las dos modalidades, se procedió a explicar el objetivo y los procedimientos del estudio. Los participantes otorgaron su consentimiento para participar de forma voluntaria a través de la firma del consentimiento informado, mismo que fue previamente aprobado por el Comité de Bioética en Investigación en el Área de la Salud de la Universidad de Cuenca (COBIAS - UC) - Ecuador (código 2019-0140EO-I). Además, a través de este documento se indicó que el proyecto garantiza el anonimato y el manejo de los datos bajo criterios éticos y deontológicos.

En cuanto a la conducción de las sesiones de los grupos focales, participamos las dos investigadoras en calidad de moderadoras. Se contó con la asistencia de cuatro ayudantes de investigación (estudiantes de últimos ciclos de la carrera de psicología; dos mujeres y dos hombres) para el registro de notas. La duración máxima de las entrevistas fue de aproximadamente $1 \mathrm{~h} 27 \mathrm{mins}$.

Todas las entrevistas fueron grabadas en audio y transcritas para su posterior análisis mediante el programa Atlas.ti. Todos los nombres utilizados en este artículo son seudónimos para garantizar el anonimato. Los datos se analizaron utilizando técnicas de codificación inductiva (Braun \& Clarke, 2006). El análisis siguió el siguiente proceso: (a) se leyó toda la información hasta tener una idea general de lo expresado por los participantes, (b) se extrajeron las unidades de significado y a su vez los temas básicos y se agruparon por códigos y (c) se crearon matrices de temas extraídos, los mismos que están respaldados por sus respectivas citas textuales.

\section{RESULTADOS Y DISCUSIÓN}

En total, nueve temas fueron identificados, mismos que se presentan a continuación.

\subsection{El feminismo como una construcción colectiva}

Las construcciones teóricas en torno al feminismo se realizaron desde dos dimensiones. Por un lado, la mayoría de participantes lo asumieron desde el ámbito colectivo: "es un movimiento social que pide el reconocimiento de los derechos de las mujeres que tradicionalmente estaban prohibidos para ellas" (Carmen, 19 años).

En estas narraciones, se reconoció un elemento transgeneracional. Se interpretó como una lucha con trascendencia y representación histórica liderada por mujeres: "fueron construyendo entre generaciones y generaciones para tratar de menorar la desigualdad" (Juan, 19 años). En Ecuador, los movimientos feministas (declarados y no declarados) surgen a principios del siglo $\mathrm{XX}$ asumiendo luchas libertarias. Las y los participantes recalcaron la búsqueda en la igualdad de derechos: "poder hacer y ser tomada en cuenta en muchos aspectos sociales, políticos, educativos, etcétera" (Hugo, 18 años), como una forma de reivindicación.

Dentro de este relato de igualdad, algunos participantes hombres hicieron alusión a las capacidades de la mujer: "[el feminismo] busca dar a conocer que las mujeres son capaces en muchas cosas más" (Antonio, 19 años). En un ejemplo dado, Juan (19 años) menciona:

[las mujeres] pueden tal vez dibujar mejor que un hombre, tal vez pueden construir mejor que un hombre, tal vez pueden ser mecánicas o ser de algún tipo de obraje que, por lo general, piensan que los hombres son mejores o se verían mejor.

Algunas de estas narraciones se enmarcaron en una argumentación esencialista asociada a la capacidad de la mujer como dadora de vida:

La mujer es mal llamada el sexo débil porque si es que ellas fueran el sexo débil, ellas no tuvieran los hijos, partiendo desde ahí [risas] (Alberto, 39 años).

La mujer como tal es dadora de vida, es un ser bastante especial, de por sí ya es fuerte (Víctor, 25 años).

Si hubiese un dios o lo que sea yo creo que sería mujer [risas] porque no hay una explicación para tanta sensibilidad o belleza que hay en la naturaleza (Miguel, 23 años).

Al respecto, Martínez (1992) plantea que, cuando existe una narrativa de carácter esencialista sobre qué es ser mujer (cualidades y atributos femeninos), esta parece contribuir con la preservación de las diferencias de género. En nuestro contexto, la maternidad es un valor cultural que destaca las expectativas sobre las mujeres relacionadas con construcciones machistas, como el marianismo (Castillo et al., 2010). Concepto que se encuentra implícito en el sistema de creencias de las y los adolescentes cuencanos (Pinos et al., 2016).

\subsection{El feminismo como una construcción individual}

Desde una segunda narrativa, únicamente algunas participantes mujeres concibieron una dimensión individual. Se refirieron al hecho de asumir conscientemente y de reestructurar, desde su espacio personal, las estructuras jerárquicas de dominación basadas en el género, en las cuales se ve inmersa la mujer.

Es un proceso de cambio interno de las mujeres, que es de cuestionarnos este orden patriarcal en el que hemos crecido, en el que hemos vivido y en el que se fundamentan nuestras relaciones y desde ahí, irlo cuestionando, ir modificando (Cecilia, 22 años).

Rosa (24 años) compartió esta percepción y expresó:

Es un despertar para las mujeres, porque quizás practicábamos y practicamos muchas normas machistas, muchos roles que nos han sido impuestos y que ni siquiera nos hemos dado en cuenta.

Respecto al hecho de ser consciente, Riquer (1992) plantea que cada mujer posee algún grado de conciencia que se traduce en autoconocimiento, el conocimiento de los otros y del mundo social en el que se encuentra inmersa $\mathrm{y}$, al desarrollar un nivel de conciencia en estos espacios la mujer existe, adquiere un sentido de yo soy (se 
reconoce) y a la vez supone un cuestionamiento de ¿qué es ser mujer en esta sociedad?

Acompañada a esta toma de consciencia, se evidenció un componente afectivo que se concibe como: "muy ligada al amor propio, al como mujer empodérate, al ámate, al que estás buenísima viéndote en un espejo [autoestima]" (Carlota, 26 años). Las participantes lo interpretaron como un proceso de amor propio, de resignificación y de sanación relacionado a su vez, con el sentido de sororidad, tanto hacia las personas que forman parte del movimiento y hacia las que no, independientemente del sexo y, que al mismo tiempo transciende las diferentes posturas que se puedan suscitar.

Ser un aliado de cualquier persona, no importa el género $y$, de alguna manera esta sororidad que podemos tener con las demás personas para visibilizar, no para ocultar, no para mezquinar, no alejar, sino más bien este sentir bonito de construir en conjunto (Rosa, 24 años).

Para Lagarde (2012), el sentido de sororidad sitúa a la mujer en una búsqueda de relaciones de carácter positivo, de establecer alianzas existenciales y de representación política; desde un cuerpo a otro, desde una subjetividad a otra, con todas esas (otras) mujeres con el fin de emprender acciones concretas para la eliminación de todas las formas de opresión.

Sin embargo, Cecilia (22 años) menciona:

Es muy complejo ser sorora con la otra persona que te han enseñado toda la vida a odiarle, a tener competencia, a sentir envidia, a tenerle celos.

La sororidad lleva implícito un nivel de complejidad que guarda relación con el proceso de ser consciente. Las relaciones entre mujeres han estado y continúan estando condicionadas por una serie de estructuras patriarcales que promueven la rivalidad.

Estas construcciones teóricas, coinciden con los planteamientos reportados por Reger (2012), en donde el feminismo se asumió como una resistencia individual y, como una acción colectiva. En efecto, en esta relación individuo - colectivo tiene lugar una importante vinculación que invita a una transformación sociocultural y a su vez, personal, que se traduce en una doble acción (Martínez, 1992).

\subsection{Tipos de feminismos}

Muy pocos participantes hicieron alusión a los tipos de feminismo. Se mencionó el feminismo socialista, el feminismo liberal y el feminismo radical. En torno a estos tipos de feminismo no se evidenció un manejo teórico, lo cual resultaba esperable, puesto que los participantes no son expertos.

...hay un feminismo socialista (Adriana, 18 años).

...este feminismo radical y este feminismo liberal de algunas mujeres (Rosa, 24 años).

La interseccionalidad supone un aspecto central que contribuye a la comprensión de la multiplicidad de movimientos. Estas diferencias radican principalmente en las agendas políticas (que deben implicar solidaridades múltiples, más allá de interposición). Por ejemplo, movimientos feministas indígenas abordan prioritariamente temas de desplazamiento territorial, conservación de los recursos naturales y la educación intercultural, mientras que, para otros movimientos feministas un aspecto central de su agenda política podría suponer el derecho al aborto (Varea, 2019). Estos objetivos responden a un contexto cultural específico y a sus necesidades reales.

Además, al feminismo radical se lo relacionó con una tendencia extremista: "el feminismo radical ha hecho que se vea como negativo" (Manuela, 23 años). Algunas participantes mujeres resaltaron la importancia de comprender la radicalidad y no limitarse únicamente a emitir un juicio de valor sin antes conocerlo. Desde un marco conceptual, el objetivo de las feministas radicales se fundamenta en descubrir y modificar la raíz de la opresión de las mujeres (cf. Friedman et al., 1987).

Hay muchas cosas radicales que no se pueden
entender y que yo tampoco lo entendía. Y de ahí
comencé a leer porque yo decía siempre tiene
que haber un por qué. Siempre hay un por qué
de las cosas, una no lo hace por loca. Entonces,
comencé a investigar y a leer un poco, te vas
empapando [enterando] de algunas cosas y vas
cambiando también esta perspectiva
[percepción de la radicalidad]. (Carlota, 26
años).

En torno a la percepción de extremismo, aparte de relacionarlo con el feminismo radical, se interpretó en términos generales: "yo si tiendo mucho a pensar en que hay, no un feminismo correcto, pero sí un feminismo verdadero" (Elena, 27 años). Al respecto, Alberto (39 años) mencionó: "el momento en que eso [feminismo] se fanatiza, automáticamente viene el caos, como tal, porque no se respetan normas, ni reglas, ni nada, sino se quiere llegar a imponer el punto a como dé lugar".

Un ejemplo de esta concepción de extremismo radica en el término hembrismo, que supone la discriminación sexual hacia el hombre, y que se ha llegado a asociar de forma errónea y directa con el feminismo: "hay esta forma de decir que el feminismo es malo porque le están confundiendo con el hembrismo" (Laura, 19 años).

\subsection{Aspectos incompatibles con el feminismo}

Durante las discusiones sobre las actitudes y pensamientos que se percibieron como incompatibles con el feminismo se mencionaron las siguientes: el patriarcado (que contempla el machismo y la violencia), el capitalismo y una actitud de superioridad y de defensividad de la mujer.

Se interpretó que el patriarcado es una estructura dominante que configura las relaciones de desigualdad en base al género: "un sistema donde el hombre es el que manda, el hombre es el que elige, el que da las decisiones" (Carlos, 18 años). Al mismo tiempo, ejerce presión en contra de los objetivos del movimiento feminista "porque quiere limitarlo por todos lados" (Rosa, 24 años). Y en efecto, tiende a invisibilizar las resistencias feministas.

Dentro del patriarcado, se mencionó: "el pensamiento machista" (Fernando, 21 años), que se asumió como un conjunto de actitudes y creencias que se sostienen en base a la percepción errónea de superioridad del hombre y, por ende, la desvalorización de la mujer. Se percibió que las conductas y pensamientos machistas están presentes tanto en hombres como en mujeres:

El hombre no es el enemigo, es el hombre con conductas machistas que no quiere verlas, 
porque el hombre con conductas machistas que está en proceso de verlas y de identificarlas y de cambiarlas, iqué bien!, porque nosotras somos mujeres también machistas, en proceso de cambiar (Cecilia, 22 años).

Igualmente, se mencionó la violencia ejercida hacia mujeres y hombres. Sin embargo, se enfatizó la vulnerabilidad del hombre. Se interpretó que, en nuestra sociedad no se visibiliza y no se brinda atención a estos casos: "vivimos en una sociedad donde la mujer es la víctima y el hombre no" (Carlos, 18 años), "sólo se busca por un lado y no se ve que también se invisibiliza mucho la violencia de las mujeres hacia los hombres o de hombres con hombres" (Manuela, 23 años). Al respecto, Hoskin et al. (2017) reportó percepciones similares, los participantes concebían que el feminismo aborda exclusivamente las necesidades de las mujeres e ignora los problemas de los hombres, al tiempo que eleva las preocupaciones de las mujeres sobre los hombres.

Sin embargo, no todas compartieron esa percepción, Rosa (24 años) manifestó:

Yo no considero que sea esta invisibilización de la violencia contra los hombres, sino más bien la violencia siempre ha estado, es algo constante y siempre las personas tratamos de normalizar esta violencia sea para hombres o mujeres, sea para quién sea de alguna manera se ha normalizado.

La violencia es estructural y su análisis se fija tanto en los cuerpos de las mujeres como feminidades normadas y en los hombres como masculinidades subordinadas $\mathrm{y}$ hegemónicas (Viteri, Ceja, \& Yépez, 2017). La interseccionalidad busca alejarse del legado de género que esencializa a mujeres y hombres, y encontrar nuevas formas de comprender las vulnerabilidades de los sujetos (Viteri et al., 2017). Un ejemplo claro lo señala Paulson (2013) quien evidencia que los hombres pobres tienen más posibilidades de morir violentamente.

Por su parte, el patriarcado y el capitalismo configuran una óptica de doble subordinación. Este último se distinguió como un sistema político que articula, fortalece y perpetúa las relaciones de desigualdad y busca un beneficio económico al apoyarse de la causa feminista: "toma a la mujer y la separa (...) se transforma en un mercado, hablando del comercio, entonces pierde su esencia [el feminismo]" (Adriana, 18 años).

Según Federici (2013), el capitalismo es un modelo alienante que ha producido la invisibilización de los trabajos que asumen la reproducción de la vida, a través de la división sexual del trabajo. Aún predomina la visión de que la mujer (por naturaleza) se ve en la obligación de responsabilizarse del espacio privado (doméstico) que ha sido concebido para ella, se acepta que asuma nuevos roles, pero sin dejar de lado los que tienen que ver con la reproducción de la vida (Groner, Muñoz \& Angulo, 2016). En el Ecuador, aunque se ha reconocido políticamente en la Constitución de la República (2008) el cuidado, poco ha significado para una mejor redistribución e implicación de los hombres (Castro-García, 2017).

En torno a la imbricación entre las relaciones sociales de reproducción y las relaciones patriarcales de género en el capitalismo, se han desarrollado distintas vertientes teóricas feministas explicativas (cf. Esquenazi, 2020).

Por último, se encontró que una actitud de superioridad y de defensiva de una mujer se percibieron como contrarias al feminismo en el sentido de que, si se busca la igualdad, la mujer no debe considerarse superior al hombre. Y, se distinguió que la mujer tiende a rechazar la ayuda del otro y la desvaloriza adoptando una postura de defensividad:

Por ejemplo, el hombre quiere ser cordial y le quiere abrir la puerta y ella [la mujer] dice "no, yo sí puedo". Pero hay veces que tienen una actitud un tanto agresiva de "iqué crees que yo no tengo la capacidad de abrir una puerta!". (Laura, 19 años).

\section{5 ¿Quiénes pueden participar del movimiento?}

Al percibir quiénes pueden formar parte o no del movimiento, las opiniones se diferenciaron en torno a dos posturas. Hay quienes concibieron que es un movimiento con representación únicamente de mujeres: "es un grupo social sólo de mujeres" (Lucía, 19 años). Además, al referirse a su participación, señalaron la importancia de la interseccionalidad, concepto que se encontraba implícito en verbalizaciones como:

Es un movimiento diverso (Juan, 19 años).

Aborda muchas culturas (Antonio, 19 años).

Cada una es en su diferencia y en su diversidad,

feminista (Cecilia, 22 años).

$\mathrm{Y}$, por otra parte, hubo participantes que lo consideraron como un movimiento abierto en el que pueden participar tanto mujeres, hombres y población LGBTI. Con respecto a la implicación de los hombres en el movimiento, se planteó que, al igual que las mujeres, es necesario un cuestionamiento del orden patriarcal, además de su situación de privilegio. Esta hace referencia a las ventajas disponibles para grupos particulares en función de los aspectos normativos de la identidad (Rothenberg, 2005).

En la actualidad, ya no es sólo la lucha de mujeres, sino unidos con hombres que ya son más pensantes, ya no tienen ese pensamiento de que ellos son superiores o de que todo gira alrededor de ellos (Hugo, 18 años).

...población LGBTI, que también son feministas (Matilde, 26 años).

En un estudio relacionado, se reportó que cuando los participantes percibían que el feminismo era sólo para mujeres, estaba presente o ausente la intención de excluir a otros grupos e identidades sociales y, por el contrario, cuando se percibía como grupo abierto se aludía a la interseccionalidad (Hoskin et al., 2017). En efecto, el feminismo debe ser interseccional para tener una relevancia, propósito e impacto (Carbado et al., 2013).

\subsection{La identificación feminista}

La identidad feminista aparece como un vasto caleidoscopio de posibles discursos y entendimientos (Munar, 2017). Dentro del continuo feminista se encontraron personas que no se identificaron como feministas, pero apoyaban su agenda (p.ej., aborto). Ello se traduce, en su diario vivir, en actitudes y acciones feministas.

Yo, por ejemplo, no me considero feminista. Yo considero que debo de tener los derechos de los seres humanos y punto. Pero, por ejemplo, una vez en casa con mi familia salió [el tema] que estoy en favor del aborto y me dijeron "pero si tienes un hijo" [y] digo, pero es que yo tuve mi hijo, yo decidí tener mi hijo, yo supe en qué 
circunstancias tuve mi hijo y ya (Elena, 27 años).

Por otro lado, estaban las mujeres que se identificaron como feministas en proceso de construcción o, a pesar de no poseer todo el conocimiento. Esta categoría no se contempla dentro del continuo feminista (soy feminista, aunque): "yo siempre digo, estoy en proceso de ser feminista" (Carlota, 26 años); "me considero feminista a pesar de que no tenga todo el conocimiento del mundo porque creo que es algo más intrínseco" (Cecilia, 22 años).

El rol del conocimiento en la predicción de la participación del feminismo no está claro y aún menos en nuestro contexto (educación formal vs. no formal). Guest (2016) plantea que el ingreso a la educación superior posibilita la participación del activismo y el desarrollar (o no) una conciencia feminista. Sin embargo, ello no supone una única forma. En este punto, tiene cabida la necesidad de cuestionar el modelo epistémico (hegemónico) de la educación formal y propiciar un diálogo intercultural. En nuestro contexto, un ejemplo claro de militancia feminista (más allá de la academia) son las diversas líderes indígenas.

Además, en torno a la identificación feminista, algunas participantes subrayaron que independientemente de auto catalogarse o no como feminista, son feministas todas aquellas personas que desde sus espacios personales emprenden acciones (p.ej., educar a los hijos) para cambiar las estructuras de opresión.

Es igual de feminista mi abuela que se quedó viuda hace más de 20 años, que tomó decisiones muy diferentes a las decisiones para su época y que es una mujer que busca su solvencia, muy fuerte, al igual que la mujer que decide estar en su casa (Cecilia, 22 años).

Es importante resaltar que la identificación feminista se ha examinado principalmente como un movimiento de identidad asociada a la pertenencia a un movimiento social feminista (Bobel, 2010). Las personas que se reconocen como feministas tienen mayor probabilidad de reconocer situaciones de sexismo y mayor participación en acciones colectivas (Munar, 2017). Sin embargo, los discursos reactivos frente al feminismo y los estereotipos negativos constituyen narrativas relacionales que limitan la voluntad de autoidentificación y a su vez, está influenciado por las experiencias personales dentro de las relaciones sociales (Dyer \& Hurd, 2016).

\subsection{Estereotipos negativos y positivos del feminismo}

$\mathrm{Al}$ abordar la percepción de los estereotipos, en un primer momento, hubo participantes que asumieron que no es posible hablar de características específicas asociadas a las personas feministas, a pesar de ser consencientes de que socialmente estos estereotipos están presentes: "yo considero también que no hay como tal una característica que defina a las personas que son feministas" (Matilde, 26 años). Sin embargo, conforme avanzaban en sus narraciones se pudo evidenciar una división muy marcada en torno a dos polos, negativo y positivo, de cómo se percibe a las personas feministas, específicamente se hizo alusión a las mujeres.

La percepción de estereotipos negativos fue la que predominó en las y los participantes. Estas percepciones se configuraron en torno al aspecto físico, a las actitudes, a estados emocionales, a la orientación sexual y a la postura religiosa. En nuestro contexto hablar de una persona feminista supone referirse a: "personas que están locas o simplemente que están equivocadas en lo que demandan" (Luis, 19 años), "las tildan de histéricas" (Antonio, 19 años), "una feminazi" (Alberto, 39 años), "exageradas" (Carlota, 26 años), "son: 'esas mujeres marimachas que no quieren trabajar', que son 'las mujeres que de verdad no tienen nada que hacer en sus casas y que por eso que están gritando en las calles" (Rosa, 24 años), "les han dicho que son lesbianas porque se juntan con mujeres" (Elena, 27 años), "se nos ha impuesto a las mujeres feministas que 'tienes que ser atea', porque es el estereotipo de feminista" (Cecilia, 22 años), "les dicen abortistas, que extremas, radicales y algunos insultos también" (Manuela, 23 años), "llevan muchos complejos y mucho dolor y no buscan la igualdad" (Elena, 27 años), "piensan que son sólo mujeres desnudas que salen a la calle a luchar por sus derechos" (Miguel, 23 años), "no muchas mujeres lo viven a profundidad sino la mayoría sólo está en eso para hacer daño o buscar como disturbios" (Fernanda, 19 años).

En otros estudios se ha encontrado que la palabra feminista conlleva connotaciones como: "odiar a los hombres", "militante", "obstinada", "enojada", "antimasculino", "agresiva", "lesbiana", "anti-madre", "poco atractiva física y sexualmente" y "extremistas radicales" (Bashir et al, 2013; Breen \& Karpinski, 2008; Rudman \& Fairchild, 2007). Específicamente, el estigma en torno a la orientación sexual (lesbianismo) puede causar reticencia a identificarse como feministas, en algunas mujeres heterosexuales (Moore \& Sthati, 2019). Asimismo, las mujeres con ideales feministas podrían distanciarse del movimiento si perciben que su entorno posee estereotipos negativos o discursos aversivos violentos (Ramsey et al., 2007).

Por su parte, la percepción de estereotipos positivos tuvo lugar en base a características físicas, actitudinales, valores morales y características a nivel cognitivo. Estas visiones fueron predominantes en las participantes mujeres: "ser fuerte, sería una mujer empoderada" (Lucía, 19 años), "un altruismo" (Adriana, 18 años), "una mujer que se respeta" (Elena, 27 años), "engloba el liderazgo, las ganas de luchar por las personas que están calladas (...), no tener vergüenza de salir a las calles a gritar, exigir derechos" (Matilde, 26 años), "una mujer independiente, una mujer que no necesite de otras circunstancias, alguien valiente" (Víctor, 25 años), "aquella que lucha por sus derechos o ve que sus derechos no sean vulnerados" (Miguel, 23 años), "ese cuestionamiento, de tal vez darse cuenta de un montón de cosas" (Cecilia, 22 años), "pacífica" (Carlos, 18).

Al respecto, Roy et al. (2007) encontraron resultados similares, los términos positivos más utilizados fueron: "independientes", "competentes", "inteligentes", "conocedoras", "fuertes" y "asertivas". Parece ser que estos estereotipos podrían influenciar aumentando el compromiso en la militancia (Moore \& Sthati, 2019).

\subsection{Percepción de factores influyentes en la connotación negativa del feminismo}

Las representaciones del feminismo son significativas tanto para la identidad feminista individual como para la percepción pública (Siegel, 2007). En nuestro contexto, se percibió que el feminismo tiene una valoración 
predominantemente negativa. Entre los factores influyentes, se hizo referencia a la educación familiar e institucional tradicional, el contexto social, la religión, la falta de empatía, la desinformación y las redes sociales, el miedo al movimiento y la existencia de un pensamiento dicotómico (distorsión cognitiva).

La familia fue vista como un espacio en donde tiene lugar la enseñanza consciente o inconsciente de los estereotipos de género $y$ que se van replicando de manera transgeneracional. Como menciona Miguel (23 años): "desde mi hogar yo me puedo dar cuenta que incluso mis familiares, en este caso personas ya de edad, abuelitas o tías abuelas, todavía tienen ese chip [mentalidad] machista, incluso ellas". Se recalcó la rigidez de pensamiento de las personas adultas, que han crecido con esos patrones cognitivos y que se asumen como verdades absolutas (distorsión cognitiva): "no podemos cambiar la ideología de las personas adultas" (Adriana, 18 años).

$\mathrm{Al}$ respecto, Alberto (39 años), percibió que el papel predominante en la educación lo asume la mujer, aludiendo a su papel de madre y cuidadora (postura esencialista):

Muchas veces, en esta sociedad, la misma mujer es la que se encarga de fomentar el machismo en las casas. Desde el punto de vista de que "no, el niño no puede lavar los platos"; "no, el niño no debe llorar" o "no, el niño esto o la niña por acá y el niño por allá”. Entonces, se van criando esas formas de pensamiento desde niños y nos van criando con esa mentalidad [machista].

Estos hallazgos coinciden con los reportados por Pinos et al. (2016), quienes evidenciaron que las y los adolescentes cuencanos perciben que los estereotipos de género se transmiten a través de la educación familiar y por la sociedad, y la mayor responsabilidad recae sobre la madre.

Por su parte, muy ligado al rol de la familia en la educación, las y los participantes mencionaron también el papel de las instituciones educativas. Se interpretó que existe un discurso contradictorio, por un lado, las instituciones educativas promulgan la igualdad, y por otro, continúan ejerciendo y promoviendo los estereotipos de género. En un ejemplo dado, Elena (27 años) expresa:

Mi hijo está en una guardería, el uniforme es gris para hombres y mujeres, pero los hombres tienen azul y las mujeres tienen rosado. Entonces, yo cuando me fui a comprar [me preguntaron] "¿para hombre o para mujer?" [y] dije: ¿no será el mismo uniforme? [me respondieron] "No, el de las mujeres tiene encaje y es rosado".

Se puede hablar de que en la misma educación que se tiene, sobre todo aquí en Cuenca, nos implantan mucho eso [estereotipos de género]. Nos implantan que "las niñas deben ser calladas", que "las niñas deben ser guardadas", "cierra tus piernas", que "no debemos andar hasta ciertas horas", que "no debemos usar cierto tipo de ropa", si es que por ahi se nos ocurre usar algo un poco provocativo que se nos acerque alguien y que nos diga algo, "es nuestra culpa", que "nosotras debemos en si estar a las órdenes, que sé yo, del papá", "hacer más caso al novio", "no tener la opción de seguir adelante” (Laura, 19 años).
La educación que recibimos desde la infancia por diversos canales pueden ser espacios de transformación, pero también de adoctrinamiento, reproducción y legitimación de procesos de socialización de género hegemónicos $\mathrm{y}$, su importancia radica en deconstruir las categorías dominantes de lo masculino y femenino (Martínez \& Ramírez, 2017). Los estereotipos de género son imaginarios culturales que están muy arraigados en nuestra sociedad.

Del mismo modo, el contexto social se percibió como conservador, tradicional y moralmente correcto. Nuestra cultura nos impone ciertos estándares que se deben cumplir: "por ejemplo, una mujer no puede ser fea como dijeron, no tiene permitido ser fea porque obviamente va a ser excluida, pero el hombre puede ser músico, puede ser escritor porque es feito, pero tiene otras cualidades" (Víctor, 25 años).

Dentro del contexto social, también se abordó el tema de la sexualización de la mujer:

Los hombres somos más instintivos e incluso vemos, yo lo hablo desde mi punto de vista no sé los hombres que estamos aquí, una modelo [chasquido de dedos] y lo primero que se nos prende es el instinto, ni siquiera decir "que guapa que está" o [ver] que ropa está usando, [chasquido de dedos] es el instinto lo primero que se nos viene. ¿Y eso por qué? lamentablemente en nuestra sociedad la mujer ha sido sexualizada, ha pasado a ser un objeto (Miguel, 23 años).

Además, hubo participantes que señalaron el papel de la religión. Se percibió que esta promueve los roles de género y promulga un estereotipo de la mujer perfecta: "se propone aquí un marianismo, este estereotipo de una mujer ideal que debe estar bajo los pies del hombre, prácticamente. No debe quejarse ante ninguna situación, aceptar todas las cosas" (Laura, 19 años).

Según la religión, yo no estoy en contra de la religión, de hecho, si soy católica, pero se dice una expresión de que "la mujer salió de la costilla del hombre”. Entonces, a veces, llega a ser un tipo de analogía errónea, en el aspecto de decir que "la mujer se debe sumir ante el hombre porque literalmente ella salió de él" (Adriana, 18 años).

En efecto, en nuestro país esta influencia es evidente, Viteri (2020) recoge una cronología de las acciones de oposición (p.ej., la campaña "con mis hijos no te metas") emprendidas por actores religiosos conservadores y que se inscriben en el ámbito público y sobre las políticas de Estado con respecto a los derechos de las mujeres (especialmente, sexuales y reproductivos).

Igualmente, la falta de empatía es un factor que cobró relevancia. Algunas participantes interpretaron que las personas no están dispuestas a realizar un ejercicio de empatía para comprender qué es lo que está pasando en la actualidad y por qué las mujeres deciden alzar sus voces, salir a las manifestaciones y reclamar sus derechos: "no se sensibilizan ante las causas" (Rosa, 24 años).

De manera análoga, se mencionó la desinformación. Existe un nivel de ignorancia frente a lo que es el feminismo y sus objetivos: "muchas de las personas ignoran de verdad las luchas, de verdad lo que está pasando" (Rosa, 24 años). Relacionado a este factor, las redes sociales contribuyen a que se propaguen discursos 
distorsionados. No promueven el desarrollo del pensamiento crítico, ya que algunas personas se limitarían únicamente a replicarlo: "es mucha ignorancia a nivel de todos los temas, no únicamente en el feminismo porque creo que en nuestra sociedad y nuestra generación sobre todo debate con meme, debate con Facebook" (Cecilia, 22 años); "justamente ven un meme, lo asocian y generalizan" (Elena, 27 años).

En conjunto, las redes sociales (p.ej., Facebook; Moore \& Stathi, 2019) son tecnologías online que proporcionan la habilidad para la construcción e interacción comunitaria (Boyd \& Ellison, 2007) que permite a las personas interactuar, crear, compartir y consumir contenido online. No obstante, cuando las discusiones que aparecen en estos medios son en base a los estereotipos de género, son a menudo, controlados o patologizados (Locke et al., 2018). Con respecto a la configuración de los memes, los investigadores identifican temas de privilegio tecnológico donde la élite masculina domina a la mujer arquetípica pasiva y sexualizada (Locke et al., 2018).

Por otro lado, se percibió que cuando las personas asumen una postura, esta suele ser rígida y dicotómica (correcto vs. incorrecto) y la misma no les permitiría analizar otros puntos de vista:

A la gente no le gusta pensar, ese es el problema, no le gusta cuestionarse las cosas, quiere las cosas ya hechas, como que esto es blanco y negro, pero entre blanco y negro hay un sinfin de tonalidades de gris (Miguel, 23 años).

Finalmente, algunas participantes mujeres se refirieron al miedo de ver a mujeres reunidas emprendiendo acciones en busca de la igualdad, como factor influyente: "les da miedo que se reúnan, lo digo así supongo y lo analizo que da miedo que se reúnan mujeres que digan jbueno ya basta!, ;se tiene que hacer algo!" (Carlota, 26 años).

\subsection{Percepción de factores influyentes en la connotación positiva del feminismo}

En la valoración positiva se encontró que los factores influyentes son principalmente la educación familiar, la aceptación y el activismo. Con respecto a la educación familiar, se observó que tiene una implicación tanto en la valoración negativa como positiva. Los participantes resaltaron la importancia de ir modificando la forma en cómo se educa (pedagogía feminista y paradigmas emancipadores) con respecto a los roles de género y con ello, la reproducción de las desigualdades.

Yo creo que entre la educación y la familia es muy importante y se han venido dando cambios muy grandes porque esto se ve reflejado en que ya no es tanto el machismo en los hombres, sino que se ha reducido en un gran porcentaje (Hugo, 18 años).

...educar a mi hijo, tratar de reeducar a mi familia, a mi medio, a mi ambiente (Elena, 27 años).

La aceptación hacia el movimiento se interpretó como un indicador de valoración positiva en términos de necesidad: "chévere que haya un grupo de personas que hacen eso porque la verdad si es que no hubiera o si es que no existiera, no se verían cambios tampoco. Yo siento que es necesario que estén" (Matilde, 26). Por su parte, se percibió que es importante la implicación directa en el movimiento: "para realmente tener una buena postura, un buen pensamiento, una buena perspectiva acerca del movimiento, se debería ser parte de él y más que todo como se maneja el movimiento" (Lucía, 19 años).

\section{CONCLUSIONES}

Algunos de los resultados aquí expuestos coinciden con los elementos teóricos encontrados en estudios previos llevados a cabo en otros contextos sobre la percepción del feminismo. Sin embargo, la investigación referente al tema en la ciudad de Cuenca es escasa y, por lo mismo, estos resultados contribuyen a comprender la complejidad existente y las características implicadas en la percepción de este constructo en el contexto socio-cultural en donde se llevó a cabo el estudio.

De los relatos de las y los participantes se puede concluir que las percepciones en torno al feminismo se configuraron desde dos esferas relacionadas; una colectiva y otra individual. A partir de la concepción colectiva, el feminismo es asumió como un movimiento social que de forma transgeneracional ha buscado el reconocimiento de los derechos de las mujeres. Sin embargo, están presentes en los participantes hombres discursos de carácter esencialista en torno a la mujer, que son el reflejo de una cultura Latinoamericana machista. Desde la perspectiva individual, se percibió el feminismo como el grado de conciencia que se asume para encontrar un sentido a la posición de la mujer en la sociedad. A su vez, esta se asoció con el sentido de amor propio, la autoestima y la sororidad, haciendo hincapié en la necesidad de reestructurar las relaciones de rivalidad entre las mujeres impuestas por el patriarcado.

En cuanto al conocimiento de los tipos de feminismo, las y los participantes percibieron su diversificación como resultado de la interseccionalidad. Hicieron únicamente alusión al feminismo socialista, liberal y radical. A este último lo relacionaron con el carácter extremista desde una connotación negativa. Como actitudes y pensamientos incompatibles con el feminismo se mencionaron el patriarcado, el machismo, el capitalismo, la violencia y una actitud de superioridad y una postura defensiva de la mujer.

Con respecto a quienes pueden participar de los movimientos feministas se presentaron dos posturas. Por una parte, se expresó que únicamente las mujeres pueden participar del movimiento haciendo nuevamente alusión a la interseccionalidad y, por otra parte, se mencionó que es un movimiento abierto a la participación de mujeres, hombres y población LGBTI, que asuman un cuestionamiento de las estructuras de dominación en la sociedad en las cuales se ven inmersas.

La identificación feminista tuvo lugar únicamente por parte de pocas participantes mujeres. La misma estuvo influenciada por la percepción de estereotipos negativos que fue predominante en las y los participantes. Estos estereotipos se configuraron en torno al aspecto físico, a las actitudes, a estados emocionales, a la orientación sexual y a la postura religiosa. Por otro lado, también tuvo lugar la percepción de estereotipos positivos que se fundamentaron en base a características a nivel cognitivo, físico, actitudinales y a valores morales. 
En la percepción de los factores influyentes en la connotación negativa del feminismo, en nuestra sociedad, se mencionaron principalmente: la educación familiar, la educación institucional, el contexto social y la religión, la falta de empatía, la desinformación y las redes sociales, el miedo al movimiento y la existencia de un pensamiento dicotómico (distorsión cognitiva). Por el contrario, los factores que parecen contribuir a una percepción positiva son la educación familiar (doble acción), la aceptación del movimiento y el activismo.

Este es un tema coyuntural a nivel nacional y si bien se han dado avances para despolarizar el rol de la mujer en la sociedad, aún se conservan estereotipos marcados en relación con el rol de la mujer. Se enfatizó la necesidad de educar en temas de género e igualdad de derechos tanto en el ámbito familiar como institucional con el fin de desarraigar el aprendizaje de las relaciones sociales basadas en la dominación y la opresión. El feminismo, en este proceso, asume un papel importante y necesario puesto que sus planteamientos rompen con estas formas relacionales disruptivas y su comprensión estaría adherida a esta reeducación.

\section{AGRADECIMIENTOS}

Este estudio fue posible gracias a la financiación de la Dirección de Investigación de la Universidad de Cuenca (Ecuador), dentro del XVIII Concurso Universitario de Proyectos de Investigación Fondos Semilla y Consolidación (Número de financiación: 20400007 2144).

\section{REFERENCIAS}

Anzoátegui, M. (2019). Desplazamientos de los discursos hegemónicos en la teoría feminista: El feminismo ecológico y animalista como nuevas perspectivas. Nomadías, 27, 33 - 50. doi:10.5354/07190905.2019.54360

Asamblea Nacional de la República del Ecuador. (2020). Texto final del Código Orgánico de Salud para la votación.

Bashir, N., Lockwood, P., Chasteen, A., Nadolny, D., \& Noyes, I. (2013). The ironic impact of activists: Negative stereotypes reduce social change influence. European Journal of Social Psychology, 43(7), 614626. doi:10.1002/ejsp.1983

Beck, M., Bryman, A. \& Futing, L. (2004). The SAGE encyclopedia of social science research methods. Thousand Oaks, CA: SAGE Publications.

Bobel, C. (2010). New blood: Third-wave feminism and the politics of menstruation. New Brunswick, $\mathrm{NJ}$ : Rutgers University Press.

Boyd, D., \& Ellison, N. (2007). Social network sites: Definition, history, and scholarship. Journal of Computer-mediated Communication, 13, 210-230. doi:10.1111/j.1083-6101.2007.00393.x

Braun, V., \& Clarke, V. (2006). Using thematic analysis in psychology. Qualitative Research in Psychology, 3(2), 77-101. doi:10.1191/1478088706qp063oa
Breen, A., \& Karpinski, A. (2008). What's in a name? Two approaches to evaluating the label feminist. Sex roles, 58, 299-310. doi:10.1007/s11199-007-9317-y

Butler, J. (2013). For white girls only? Post feminism and the politics of inclusion. Feminist Formations, 25, 35-58. doi:10.1353/ff.2013.0009

Carbado, D., Crenshaw, K., Mays, V., \& Tomlinson, B. (2013). Intersectionality: Mapping the movements of a theory. Du Bois Review: Social Science Research on Race, 10(2), 303-312. doi:10.1017/S1742058X13000349

Carey, M., \& Asbury, J. (2012). Focus group research. Walnut Creek, CA: Left Coast Press.

Castillo, L., Perez, F., Castillo, R., \& Ghosheh, M. (2010). Construction and initial validation of the Marianismo beliefs scale. Counselling Psychology Quarterly, 23(2), 163-175. doi:10.1080/09515071003776036

Castro-García, C. (2017). Políticas para la igualdad. Madrid, España: Los libros de la catarata

Collins, P. (2000). Black feminist thought: Knowledge, consciousness, and the politics of empowerment. Abingdon, UK: Routledge.

Díaz, G. (2005). Los grupos focales. Su utilidad para el médico de familia. Revista Cubana de Medicina General Integral, 21(3), 1-9.

Díaz, V. (08 de marzo de 2018). 'Desenmascarar' al feminismo. El Comercio. Recuperado de https://www.elcomercio.com/afull/genero-feminismodiainternacionaldelamujer-mitos-experimental.html

Dyer, S., \& Hurd, F. (2016). Changing perceptions about feminists and (still not) claiming a feminist identity. Gender and Education, 30(4), 435-449. doi:10.1080/09540253.2016.1216524

Esquenazi, A. (2020). Relaciones sociales de producción y relaciones patriarcales de género en el capitalismo: una mirada más allá del aparente dualismo. Revista Marx e o Marxismo, 8(4), 72-92.

Federici, S. (2013). Revolución en punto cero. Trabajo doméstico, reproducción y luchas feministas. Madrid, España: Traficantes de Sueños.

Friedman, M., Metelerkamp, J., \& Posel, R. (1987). What Is Feminism? Agenda, Empowering Women for Gender Equity, 1, 3-24.

doi:10.1080/10130950.1987.9674671

Gamba, S. (2007). Diccionario de estudios de género y feminismos. Buenos Aires, Argentina: Editorial Biblos.

García, M., Alvira, F., \& Alonso, L. (2015). El análisis de la realidad social. Métodos y técnicas de investigación ( $4^{\mathrm{a}}$ ed.). Madrid, España: Alianza editorial.

García, C., \& Valdivieso, M. (2005). Una aproximación al movimiento de mujeres en América Latina: De los grupos de autoconciencia a las redes nacionales y trasnacionales. Buenos Aires, Argentina: Consejo Latinoamericano de Ciencias Sociales.

Gifford, D. (2011). Show or tell? Feminist dilemmas and implicit feminism at girls' rock camp. Gender y Society, 25(5), 569-588. doi:10.1177/0891243211415978

Groner, C., Muñoz, E., \& Angulo, V. (2016). La percepción de la Imagen de la Mujer en los medios de comunicación social en el Ecuador. Quito, Ecuador: Consejo de Regulación y Desarrollo de la Información y Comunicación. 
Guest, C. (2016). Knowing feminism: the significance of higher education to women's narratives of 'becoming feminist', Gender and Education, 28(3), 471-476. doi:10.1080/09540253.2016.1167842

Hemmings, C. (2012). Affective solidarity: Feminist reflexivity and political transformation. Feminist Theory 13(2), 147-161. doi: $10.1177 / 1464700112442643$

Hopkins, P. (2017). Social geography I: Intersectionality. Progress in Human Geography, 43(5), 937-947. doi:10.1177/0309132517743677

Hoskin, R., Jenson, K., \& Blair, K. (2017). Is our feminism bullshit? The importance of intersectionality in adopting a feminist identity. Cognitive Social Sciences, 3, 1-19. doi:10.1080/23311886.2017.1290014

Jaggar, A. (1983). Feminist politics and human nature. Lanham, MD: Rowman y Littlefield Publishers

Jolles, M. (2012). Going rogue: Post feminism and the privilege of breaking rules. Feminist Formations, 24(3), 43-61. doi:10.1353/ff.2012.0031

Lagarde, M. (2014). Pacto entre mujeres. Sororidad. En M. Lagarde (Ed.), El feminismo en mi vida. Hitos, claves y utopías (pp. 557-569). México: Coordinación de relaciones públicas del Instituto de las mujeres del Distrito Federal.

Locke, A., Lawthom, R., \& Lyons, A. (2018). Social media platforms as complex and contradictory spaces for feminism: Visibility, opportunity, power, resistance and activism. Feminism \& Psychology, 28, 3-10. doi:10.1177/0959353517753973

López, M. Lerendegui, M., Simon, T., Aznar, C., Martin, D., Briz, T., \& Botaya, R. (2011). Una mirada al género en la investigación, análisis cualitativo. Atención Primaria, 43(10), 531-535. doi:10.1016/j.aprim.2010.09.018

Katzenstein, M. (1987). Comparing the feminist movements of the United States and Western Europe An overview. In M. Katzenstein y C. Mueller (Eds.), The women's movements of the United States and Western Europe (pp. 3-22). Philadelphia, PA: Temple University Press.

Martin, P. (1990). Rethinking feminist organizations. Gender \& society, 4(2), 182-206. doi:10.1177/089124390004002004

Martínez, A. (1992). La identidad femenina: crisis y construcción. En M. Tarrés (Ed.), La voluntad del Ser (pp. 65-84). México, DF: Colegio de México.

Martínez, I., \& Ramírez, G. (2017). Des-patriarcalizar y des-colonizar la educación. Experiencias para una formación feminista del profesorado. Revista Internacional de Educación para la Justicia Social, 6(2), 81-95. doi:10.15366/riejs2017.6.2.005

Montero, J. (2006). Feminismo: un movimiento crítico. Intervención Psicosocial, 15(2), 167-180.

Moore, A., \& Stathi, S. (2019). The impact of feminist stereotypes and sexual identity on feminist selfidentification and collective action. The Journal of Social Psychology, 160(3), 267-281. doi:10.1080/00224545.2019.1644280

Munar, A. (2017). To be a feminist in (tourism) academia. Anatolia, 28(4), 514-529. doi:10.1080/13032917.2017.1370777

Muñoz, L. (15 de mayo de 2018). Ni machismo ni feminismo. El Mercurio. Recuperado de https://ww2.elmercurio.com.ec/2018/05/15/nimachismo-ni-feminismo/
Mueller, C. (1987). Collective consciousness, identity transformation, and the rise of women in public office in the United States. In M. Katzenstein y C. Mueller (Eds.), The women's movements of the United States and West Europe. Philadelphia, PA: Temple University Press.

Myers, G. (1998). Displaying opinions: topics and disagreement in focus groups. Language in Societv, 27, 85-111.

Onwuegbuzie, A., Dickinson, W., Leech, N., \& Zoran, A. (2011). Un marco cualitativo para la recolección y análisis de datos en la investigación basada en grupos focales. Paradigmas, 3, 127-157

Organización de las Naciones Unidas. (2015). Transformar nuestro mundo: la Agenda 2030 para el Desarrollo Sostenible.

Pardo, R. (2019). Avances y retos de las políticas de igualdad y del feminismo en Ecuador. Revista científica de la universidad del Pacífico, 7, 17-31. doi:10.35936/caracter.v7i1.54

Paulson, S. (2013). Masculinidades en movimiento: transformación territorial y sistemas de género. Buenos Aires, Argentina: Teseo.

Pinos, V., Pinos, G., Baitar, R., Jerves, M., \& Enzlin, P. (2016). Perception of gender stereotypes, machismo and manianismo in Ecuadorian adolescents: A focus group study. Maskana, 7(2), 17-28. doi:10.18537/mskn.07.02.02

Ramsey, L., Haines, M., Hurt, M., Nelson, J., Turner, D., Liss, M., \& Erchull, M. (2007). Thinking of Others: Feminist identification and the perception of others' beliefs. Sex Roles, 56(9), 611-616. doi:10.1007/s11199-007-9205-5

Reger, J. (2012). Everywhere and nowhere: Contemporary feminism in the United States. Oxford, UK: Oxford University Press.

Riquer, F. (1992). La identidad femenina en la frontera entre la conciencia y la interacción social. En M. Tarrés (Ed.), La voluntad de ser. Mujeres en los noventa (pp. 51-64). México: El Colegio de México

Rothenberg, P. (2005). White privilege: Essential readings on the other side of racism $\left(2^{\mathrm{a}} \mathrm{ed}\right.$.). New York, NY: Worth Publishers.

Roy, R., Weibust, K., \& Miller, C. (2007). Effects of stereotypes about feminists on feminist selfidentification. Psychology of Women Quarterly, 31(2), 146-156. doi:10.1111/j.1471-6402.2007.00348.x

Rudman, L., \& Fairchild, K. (2007). The F word: Is feminism incompatible with beauty and romance? Psychology of Women Quarterly, 31(2), 125-136. doi:10.1111/j.1471-6402.2007.00346.x

Scharager, J., \& Reyes, P. (2001). Muestreo no probabilístico. Pontificia Universidad Católica de Chile, Escuela de Psicología, 1-3.

Secretaría Nacional de Planificación y Desarrollo. (2017). Plan Nacional de Desarrollo 2017-2021. Quito, Ecuador: Dirección de Comunicación Social.

Siegel, D. (2007). Sisterhood interrupted: From radical women to girls gone wild. New York, NY: Palgrave Macmillan.

Taylor, S. \& Bogdan, R. (1992). La implementación de los procesos de investigación social cualitativos. Barcelona, España: Paidós. 
Valdivieso, M., Girón, A., Vasallo, N., Sagot, M., Carosio, A., González, M... \& Correa, E. (2012). Feminismo y cambio social en América Latina y el Caribe. Buenos Aires, Argentina: Consejo Latinoamericano de Ciencias Sociales.

Valentine, G. (2007). Theorizing and researching intersectionality: A challenge for feminist geography. The Professional Geographer, 59, 10-21. doi:10.1111/j.1467-9272.2007.00587.x
Varea, S. (2019). Feminismos entrecruzados: luchas por la despenalización del aborto y medioambiente en el Ecuador. Ciencia Política, 14(27), 207-226.

Viteri, M. (2020). Políticas antigénero en América Latina: Ecuador. Río de Janeiro, Brasil: Observatorio de sexualidad y política.

Viteri, M., Ceja, I., y Yépez, C. (2017). Corpografías: género y fronteras en América Latina. Quito, Ecuador: FLACSO. 